\title{
Compositional persistence in a multicyclic network of synthetic replicators
}

\author{
Jürgen Huck, Tamara Kosikova, and Douglas Philp*
}

\author{
School of Chemistry and EaStCHEM, University of St Andrews, \\ North Haugh, St Andrews, Fife KY16 9ST, UK
}

${ }^{*}$ Corresponding author: d.philp@st-andrews.ac.uk

\begin{abstract}
The emergence of collections of simple chemical entities that create self-sustaining reaction networks, embedding replication and catalysis, are cited as potential mechanisms for the appearance on the early Earth of systems that satisfy minimal definitions of life. In this work, a functional reaction network that creates and maintains a set of privileged replicator structures through auto- and cross-catalyzed reaction cycles is created from the pairwise combinations of four reagents. We show that the addition of individual pre-formed templates to this network, representing instructions to synthesize a specific replicator, induces changes in the output composition of the system that represent a network-level response. Further, we establish through sets of serial transfer experiments that the catalytic connections that exist between the four replicators in this network and the system-level behavior thereby encoded impose limits on the compositional variability that can be induced by repeated exposure to instructional inputs, in the form of preformed templates, to the system. The origin of this persistence is traced through kinetic simulations to the properties and inter-relationships between the critical ternary complexes formed by the auto- and crosscatalytic templates. These results demonstrate that in an environment where there is no continuous selection pressure, the network connectivity, described by the catalytic relationships and system-level interactions between the replicators, is persistent, thereby limiting the ability of this network to adapt and evolve.
\end{abstract}




\section{Introduction}

The emergence of life on Earth signaled the appearance of self-sustaining systems that could harness non-linear processes in the pursuit of complex functions, such as replication, self-sorting, ensemble-based control mechanisms and, ultimately, chemical evolution. It has been suggested ${ }^{1}$ that small organic molecules could create functional networks through sets of auto- and cross-catalyzed reaction cycles that, in turn, could select and amplify favored components. This process would lead to the appearance of sets of privileged molecular structures that are persistent within these networks. The mechanisms by which these networks could emerge are the subject of significant debate $^{2}$ and developing an understanding of processes that can transition groups of simple chemical entities into more complex systems is a key target for the emerging field ${ }^{3}$ of systems chemistry.

Minimal replication processes are often placed ${ }^{2 \mathrm{~d}, \mathrm{e}, 3 \mathrm{a}, \mathrm{f}}$ at the center of these transitions and, consequently, may represent an important requirement for the appearance on Earth of systems that satisfy minimal definitions ${ }^{4}$ of life. Therefore, the emergence of persistent sets of molecules that can establish and manage replication within small chemical networks is a critical prerequisite for the appearance of such systems. Experimental synthetic replicating systems, ${ }^{5}$ developed by us $^{6}$ and others, ${ }^{7-9}$ have demonstrated that template-driven replication is possible in synthetic systems using a variety of chemistries: from oligonucleotides ${ }^{7}$ and peptides $^{8}$ to small organic molecules. ${ }^{6,9}$ In isolation, individual replicators behave in predictable ways and are capable of processing ${ }^{10}$ pools of reagents to direct and amplify their own formation. However, the presence of several interconnected catalytic processes within the same reaction network can give rise to significantly more complex phenomena, such as

programmed outputs, ${ }^{6 b, 8 e, 11}$ feedback loops ${ }^{12}$ and oscillations. ${ }^{13}$ In the context of "metabolism-first" scenarios ${ }^{1,2 \mathrm{~d}}$ for the emergence of life, network regulation in terms of composition is required to ensure the persistence of the key chemical constitutions that sustain the network. While taking inspiration from the complexity of natural systems, we wish to explore the persistence ${ }^{14}$ of replicators in networks through a system that possesses both structural and interactional simplicity in terms of its chemical 
components. Such systems can be analyzed and characterized comprehensively, thereby providing a methodological grounding for the development and understanding, both experimental and theoretical, of the processes relevant to the origin of life.

A prerequisite for examining replicator persistence is a network of interconnected and interdependent replication cycles in which the replicating templates compete with each other for the chemical building blocks necessary for their construction. The building blocks themselves must possess recognition sites that can be used to direct the requisite replication processes and reaction sites that can deliver a programmed pattern of reactivity. To this end, we identified a set of four reagents, shown at the center of Figure 1. These four reagents, $\mathbf{A}$ to $\mathbf{D}$, are grouped such that each individual reagent possesses one of two possible recognition sites and one of two possible reactive elements. Thus, reagent $\mathbf{A}$ and reagent $\mathbf{B}$ possess recognition sites and reactive elements that are complementary to each other and their combination gives rise to selfreplicator T1, which is capable of directing its own formation through an autocatalytic cycle (Figure 1a, top left). Reagents $\mathbf{C}$ and $\mathbf{D}$ have a similar relationship and afford selfreplicator T2, which is also capable of directing its own formation through an autocatalytic cycle (Figure 1a, bottom right). By contrast, reagents $\mathbf{B}$ and $\mathbf{C}$ possess complementary reactive elements, but identical recognition sites. The combination of these two reagents affords template R1 (Figure 1a, top right), which bears identical recognition sites and so cannot catalyze its own formation directly. Similarly, reagents $\mathbf{A}$ and $\mathbf{D}$ also possess complementary reactive elements, but identical recognition sites and their combination affords template R2 (Figure 1a, bottom left), which also cannot catalyze its own formation directly. However, taken together, R1 and R2 are mutually complementary in terms of recognition and can therefore participate (Figure 1a, top right and bottom left) in two crosscatalytic cycles whereby $\mathbf{R} \mathbf{1}$ catalyzes the formation of $\mathbf{R 2}$ and vice versa. These relationships represent a reciprocal replication cycle. 
(a)

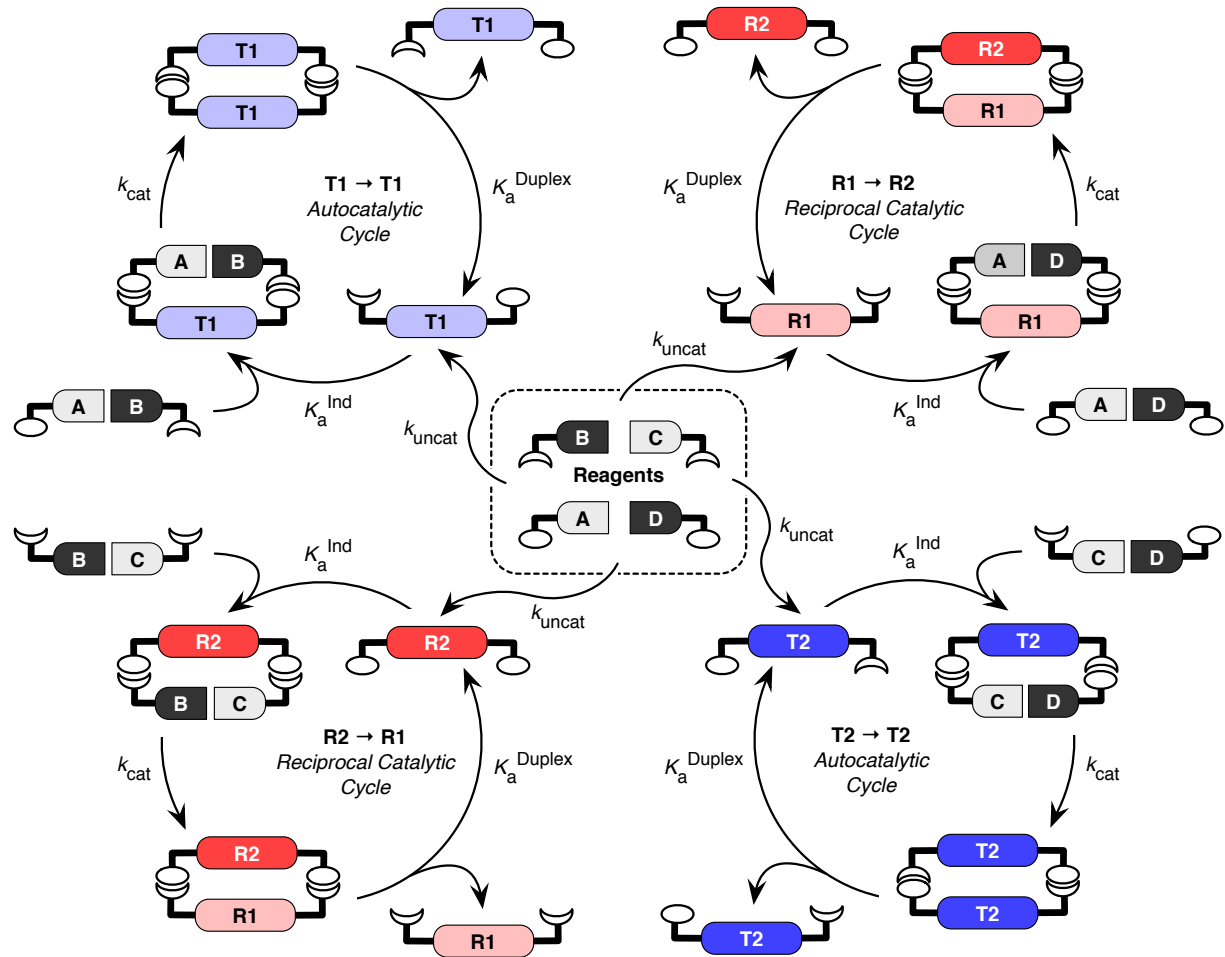

(b)
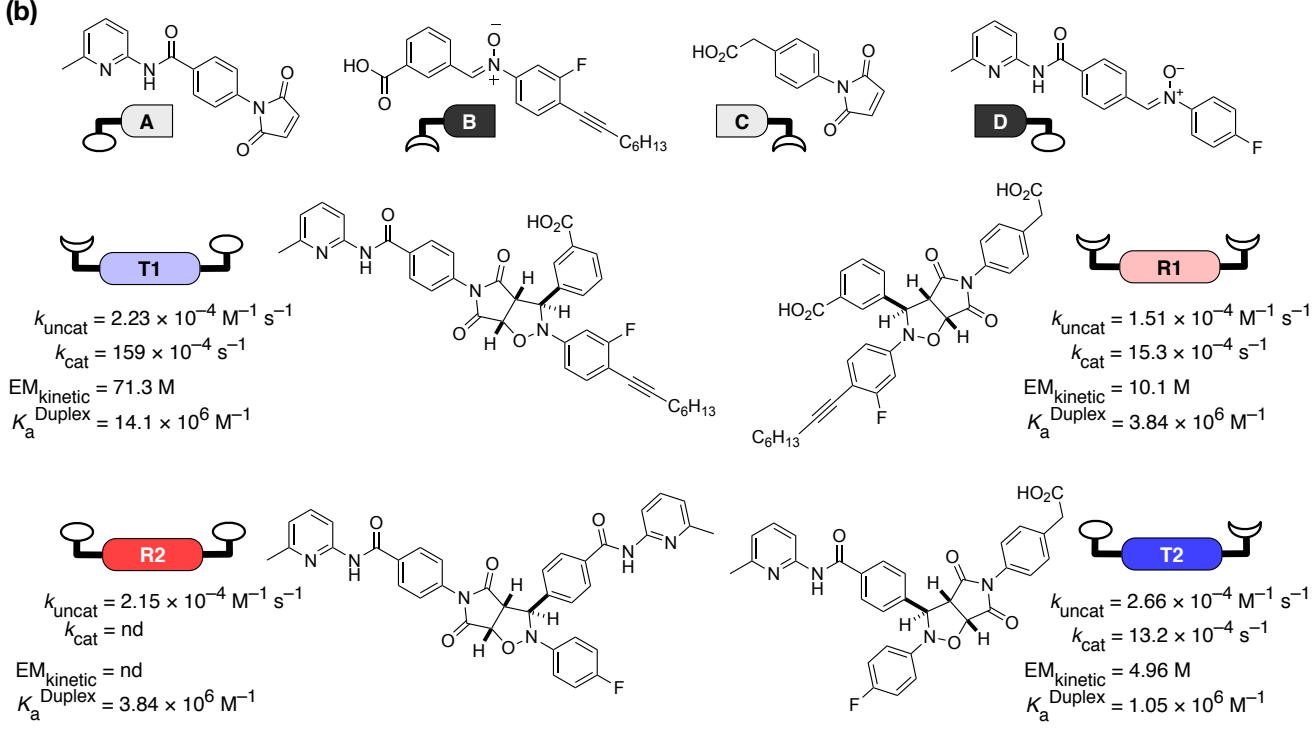

Figure 1. Reagents $\mathbf{A}$ to $\mathbf{D}$ react to create a multicyclic network of interdependent replicators. (a) Pairwise reactions of four reagents (A to $\mathbf{D}$, center) form four replicators T1, T2, R1 and R2 through bimolecular pathways ( $k_{\text {uncat }}$ ). Minimal replicators T1 and T2 are capable of assembling the reagents required to copy themselves and accelerating the reaction between them $\left(k_{\text {cat }} ; \mathrm{EM}_{\text {kinetic }}=k_{\text {cat }} / k_{\text {uncat }}\right)$, completing autocatalytic cycles (top left and bottom right). Reciprocal replicators $\mathbf{R} 1$ and $\mathbf{R 2}$ are capable of assembling the reagents required to create their complementary partner and then accelerating the reaction between them $\left(k_{\mathrm{cat}}\right)$, completing reciprocal catalytic cycles (top right and bottom left). (b) Chemical structures of reagents $\mathbf{A}$ to $\mathbf{D}$ and the replicators (T1, T2, R1 and R2) derived from these reagents. Key kinetic and thermodynamic parameters for each replicator are given next to the relevant structure. nd indicates parameters that could not be determined experimentally as a result of the limited solubility of $\mathbf{R 1}$ in $\mathrm{CDCl}_{3}$ following its purification. 
The network shown in Figure 1a possesses the minimal lexicon of intermolecular interactions and reactions required to create a tightly coupled reaction network that contains both self-replicators (autocatalytic, T1 and T2) and reciprocal replicators (cross-catalytic, R1 and R2). Since these replicators must construct themselves from a common reagent pool (A to $\mathbf{D}$ ), we expect that the product distribution expressed by this network will be amenable to perturbation by the addition ${ }^{5,6}$ of the instructional templates (T1, T2, R1 and R2).

In order to implement this network experimentally, it is necessary to design suitable building blocks to assume the roles of reagents $\mathbf{A}$ to $\mathbf{D}$. To this end, we exploited our previous work in the design and construction of both self- and reciprocal replicators $^{6,10,15}$ to identify the four compounds shown in Figure $1 \mathrm{~b}$. This group of compounds contains two maleimides ( $\mathbf{A}$ and $\mathbf{C}$ ) and two nitrones (B and $\mathbf{D})$. Pairwise 1,3-dipolar cycloaddition reactions afford two self-replicators, T1 and T2, and two reciprocal replicators, R1 and $\mathbf{R 2}$. A key consideration in the design of the network components is the ease with which the composition of the reaction mixture can be determined experimentally. To this end, a fluorine atom is present in both $\mathbf{B}$ and $\mathbf{D}$ and, thus, a fluorine atom is also present in each of the templates, allowing ${ }^{19} \mathrm{~F}\left\{{ }^{1} \mathrm{H}\right\} N M R$ spectroscopy to be used as the analytical tool for this network. The large dispersion in ${ }^{19} \mathrm{~F}$ chemical shifts ensures that all of the critical compounds present in the network can be identified and quantified unambiguously.

In this work, we describe the properties of the tightly coupled reaction network built from this set of simple synthetic replicators and demonstrate that the population distribution within this network can be influenced in predictable ways by the addition of replicators as instructions. The system-level ${ }^{16}$ properties expressed by this network are such that the global population ratios of the replicators within the network are resistant to changes brought about by external pressure. This resistance is demonstrated to be a consequence of the catalytic relationships encoded by the network. 


\section{Results and Discussion}

\section{Network characterization}

Initially, we characterized the replicating behavior of each of the individual templates experimentally and used published protocols $s^{6,10,15}$ for the analyses of experimental data from replicators to extract the key kinetic and thermodynamic parameters for each template (Figure 1b; for details, see the Supporting Information). Having characterized the replicators in isolation, we next assessed the performance of the complete network. A solution of reagents $\mathbf{A}$ to $\mathbf{D}$ in $\mathrm{CDCl}_{3}([\mathbf{A}]=[\mathbf{B}]=[\mathbf{C}]=[\mathbf{D}]=10 \mathrm{mM})$ was prepared and the formation of the replicators, $\mathbf{T} 1, \mathbf{T 2}, \mathbf{R} 1$ and $\mathbf{R} 2$ was monitored by $470 \mathrm{MHz}{ }^{19} \mathrm{~F}\left\{{ }^{1} \mathrm{H}\right\}$ NMR spectroscopy (Figure 2a) over a period of 40000 s. From the concentration-time profiles for the species present in the network (Figure 2a), it is clear that the rates of formation of the reciprocal replicators, $\mathbf{R 1}$ and $\mathbf{R 2}$, are higher than those for the selfreplicators, T1 and $\mathbf{T 2}$, and that the rates of formation within each class of replicators are tightly coupled. These differential reaction rates for the two classes of replicators result in a 1.9:1 preference (Figure 2b, No T) for the formation of R1 and R2 over T1 and T2.

In general, the introduction of a self-replicating template to a reaction mixture represents $^{5,6}$ an instruction to up-regulate the production of the added template. Similarly, the introduction of a reciprocal replicating template to a reaction mixture represents an instruction to up-regulate the production of the replicator that is complementary to the added template. In order to investigate the ability of a templatebased instruction to alter the output of our replicator network, we performed a series of experiments in which a solution of the reagents $\mathbf{A}$ to $\mathbf{D}$ in $\mathrm{CDCl}_{3}([\mathbf{A}]=[\mathbf{B}]=[\mathbf{C}]=[\mathbf{D}]=$ $10 \mathrm{mM}$ ) was instructed by the addition of $20 \mathrm{~mol} \%$ of either R2, T1 or T2 at $t=0$. The concentrations of the four replicators, T1, T2, R1 and R2 were then determined by $470 \mathrm{MHz}{ }^{19} \mathrm{~F}\left\{{ }^{1} \mathrm{H}\right\}$ NMR spectroscopy after a period of $40000 \mathrm{~s}$. These experiments (Figure $2 b$ ) reveal significant changes in the concentrations of all of the replicators in each experiment compared to that where no replicator instruction has been added. 


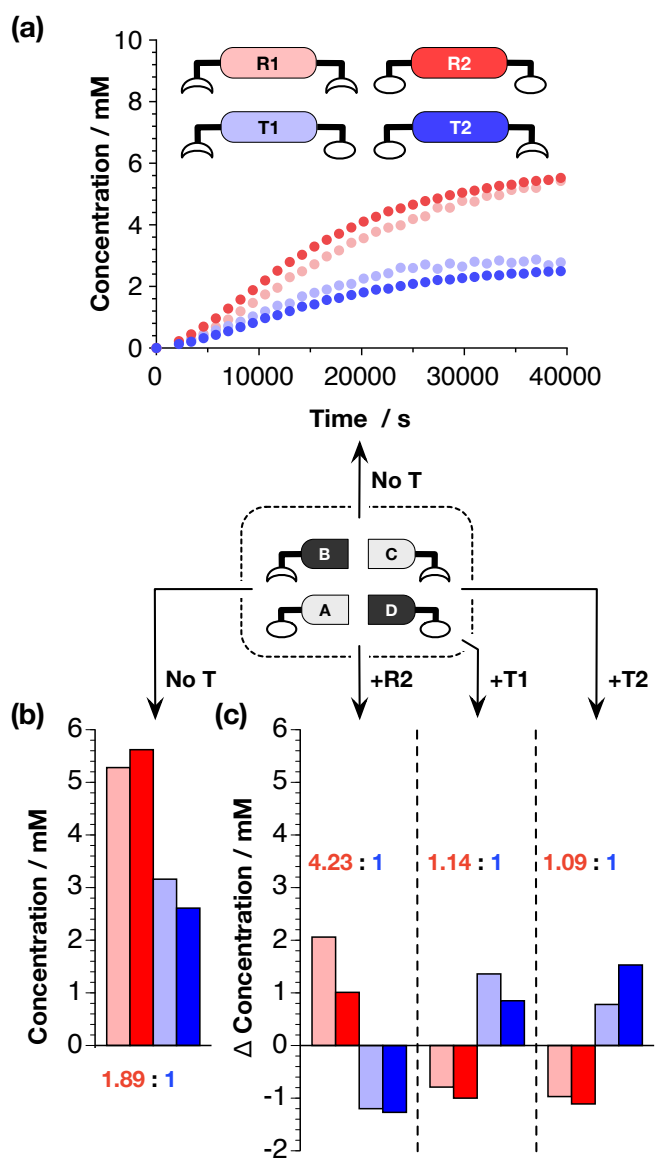

Figure 2. Individual replicators act as instructions to the multicyclic network. (a,b) In the absence of an instructional template, reactions of $\mathbf{A}$ to $\mathbf{D}$ within the network shown in Figure $1([\mathbf{A}]=[\mathbf{B}]=[\mathbf{C}]=[\mathbf{D}]=10$ $\mathrm{mM}$ in $\mathrm{CDCl}_{3}$ at $283 \mathrm{~K}$, monitored by $470 \mathrm{MHz}{ }^{19} \mathrm{~F}\left\{{ }^{1} \mathrm{H}\right\}$ NMR spectroscopy) result in the formation of a mixture of replicators. The combined concentrations of reciprocal replicators (R1 and R2, pink and red circles (a) and pink and red bars (b)) are approximately twice those of the autocatalytic replicators (T1 and T2, light and dark blue circles (a) and light and dark blue bars (b)). (c) The addition of specific replicators, introduced as network instructions, at the start of the reaction between reagents $\mathbf{A}$ to $\mathbf{D}$ elicits both predictable and system-level changes in the composition of the reaction network after $40000 \mathrm{~s}$ $(11.1 \mathrm{~h})$. The addition of a reciprocal replicator (+R2) increases the concentrations of both reciprocal replicators and the addition of one autocatalytic replicator (either $+\mathbf{T 1}$ or $+\mathbf{T 2}$ ) increases the concentrations of both autocatalytic replicators. The poor solubility of $\mathbf{R} \mathbf{1}$ in $\mathrm{CDCl}_{3}$ following its purification precluded its use as instruction in template-directed experiments.

When the network is instructed by the addition of $\mathbf{R 2}$, the preference for the formation of the reciprocal replicators overall is increased to 4.2:1. However, the replicator that is up-regulated most strongly is, in fact, R1, i.e., the complementary partner of the instructing template $\mathbf{R 2}$, reflecting the instruction given to the system-make $\mathbf{R} \mathbf{1}$. The reciprocal, crosscatalytic relationship between $\mathbf{R} 1$ and $\mathbf{R 2}$ ensures that the increase in production of $\mathbf{R} \mathbf{1}$ arising from the presence of the $\mathbf{R} \mathbf{2}$ instruction also results in an 
increase in the concentration of the instructing template itself. By contrast, when the network is instructed by the addition of self-replicator $\mathbf{T 1}$, the preference for the formation of the reciprocal replicators is erased almost entirely - the ratio of the reciprocal replicators (R1 and $\mathbf{R 2}$ ) to the self-replicators (T1 and T2) is now 1.1:1. In this case, however, the replicator that is up-regulated the most is the instructing template T1 itself, reflecting the fact that this template is capable of directing its own formation only. Similar results are obtained when the network is instructed with self-replicator T2-the ratio of the reciprocal replicators (R1 and $\mathbf{R 2}$ ) to the self-replicators (T1 and T2) is also 1.1:1.

The effects of instruction are also reinforced by the connectivity of the reaction network created by the four replicators. For example, instructing the system with T1 up-regulates not only the production of this self-replicator within the network, but also the other self-replicator T2. This observation is, at first sight, odd, since there is no explicit cross-catalytic relationship between T1 and T2. However, this system-level effect can be understood readily by considering the effect of the T1 added on the rates of the various reaction processes that form the replicators within the network. Selfreplicator $\mathbf{T} \mathbf{1}$ is constructed from building blocks $\mathbf{A}$ and $\mathbf{B}$ and the addition of $\mathbf{T} \mathbf{1}$ at the start of the experiment will increase the initial rate of their consumption. In the absence of appropriate added templates, the other replicators, T2, R1 and R2, all rely on bimolecular reactions to form either themselves or their complementary partner before the respective catalytic cycles can become effective. The reciprocal replicators $\mathbf{R} \mathbf{1}$ and $\mathbf{R} 2$ require building blocks $\mathbf{A}$ and $\mathbf{B}$, respectively. Since these two building blocks are consumed at significantly increased initial rates in the presence of added $\mathbf{T} 1$ through the autocatalytic cycle that T1 exploits to form itself, the bimolecular rates for the formation of R1 and R2 are decreased significantly. By contrast, self-replicator T2 actually benefits from these changes in the rates of formation of $\mathbf{R 1}$ and $\mathbf{R 2}$, since T2 requires building blocks $\mathbf{C}$ and $\mathbf{D}$ only. Consequently, T2 will be formed at a comparatively higher initial rate than either $\mathbf{R} \mathbf{1}$, which requires $\mathbf{B}$, or $\mathbf{R 2}$, which requires A. Therefore, the reagent flux through the autocatalytic cycle involving T2 becomes significant at an earlier time point than the cross-catalytic cycles involving the reciprocal replicators $\mathbf{R} 1$ and $\mathbf{R 2}$. Since $\mathbf{R} 1$ and $\mathbf{R} 2$ also require building blocks $\mathbf{C}$ and $\mathbf{D}$ for their 
formation, this small advantage afforded to T2 through the differences in the bimolecular rates is amplified by the autocatalytic formation of T2 and results in the observed up-regulation $\mathbf{T 2}$ at the expense of $\mathbf{R} \mathbf{1}$ and $\mathbf{R 2}$.

\section{Replicator persistence within the network}

This replicator network shows both predictable responses to direct template inputs and exhibits system-level behavior that arises as a result of interconnections between the species within it. These features would lead to the appearance of sets of privileged molecular structures that are persistent within these networks. The mechanisms by which these networks could emerge are the subject of significant debate ${ }^{2}$ and developing an understanding of processes that can transition groups of simple chemical entities into more complex systems is a key target for the emerging field ${ }^{3}$ of systems chemistry. On the early Earth, such networks would have been subject ${ }^{17}$ to pressures from compositional changes in the reagent feed and environmental changes. These pressures would have challenged the stability and persistence of potentially fragile replicator networks. In order to establish experimentally the effect of such events on the replicator composition within this network, we designed a set of serial transfer experiments $^{18}$ (Figure 3 ) that subjected the replicator network to simulated environmental changes.

Firstly, we wished to explore how our network responded to environmental events in which a fresh input of the starting materials only was provided. The intrinsic kinetic properties of the network generate an approximately 2:1 preference for the reciprocal replicators $\mathbf{R} \mathbf{1}$ and $\mathbf{R 2}$, and we wished to explore whether this natural bias was stable under the environmental conditions described. Accordingly, we performed an initial experiment in which a solution of the reagents $\mathbf{A}$ to $\mathbf{D}$ in $\mathrm{CDCl}_{3}$ was prepared $([\mathbf{A}]=[\mathbf{B}]=$ $[\mathbf{C}]=[\mathbf{D}]=10 \mathrm{mM}$, Figure $3 \mathrm{a}$, box labeled F). No additional instructional template was added to this solution at the start of the reaction. The formation of the minimal replicators, $\mathbf{T} 1$ and $\mathbf{T 2}$, and the reciprocal replicators, R1 and R2 was assayed by $470 \mathrm{MHz}{ }^{19} \mathrm{~F}\left\{{ }^{1} \mathrm{H}\right\}$ NMR spectroscopy after complete consumption of starting materials. 
(a)

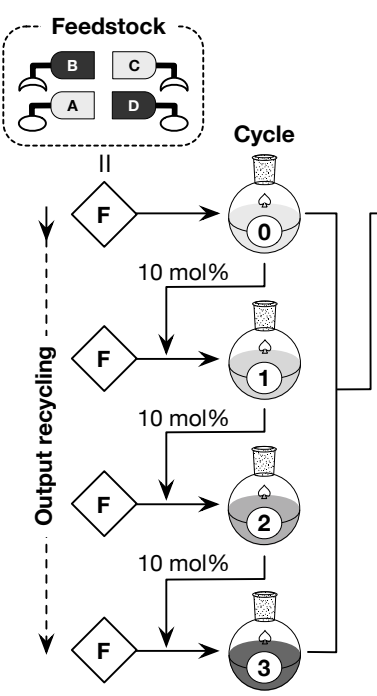

(b)
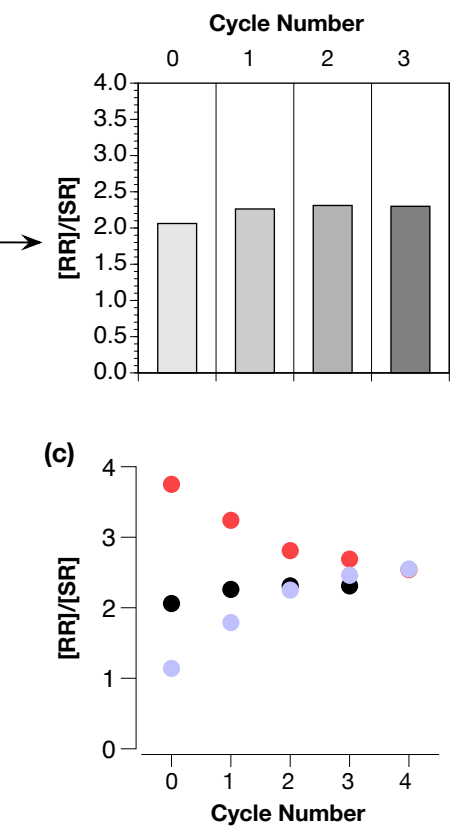

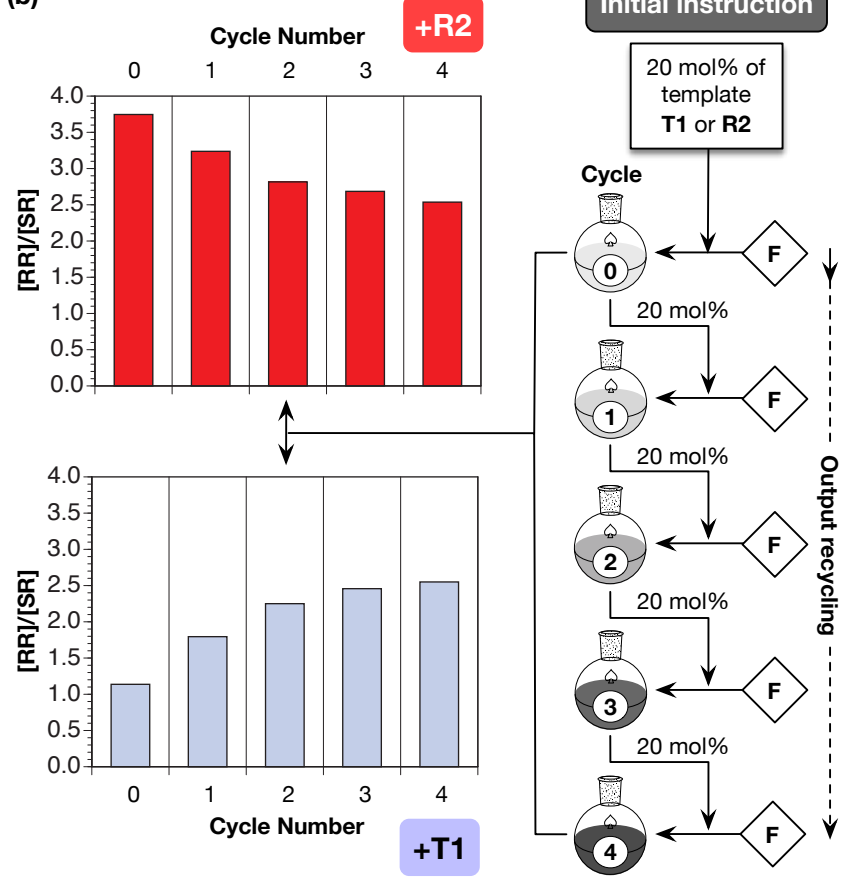

Figure 3. A set of serial transfer experiments demonstrates replicator persistence within the multicyclic network. The outcome of a reaction between reagents $\mathbf{A}$ to $\mathbf{D}\left([\mathbf{A}]=[\mathbf{B}]=[\mathbf{C}]=[\mathbf{D}]=10 \mathrm{mM}\right.$ in $\mathrm{CDCl}_{3}$ at $283 \mathrm{~K}$, monitored by $470 \mathrm{MHz}{ }^{19} \mathrm{~F}\left\{{ }^{1} \mathrm{H}\right\}$ NMR spectroscopy) can be used as the instructional input for a subsequent reaction. (a) In the absence of an initial instruction, the ratio [RR]/[SR] $(=([\mathbf{R 1}]+[\mathbf{R 2}]) /([\mathbf{T} 1]+[\mathbf{T 2}]))$ increases slightly across four cycles from 2.06 to 2.30 when 10 mol\% of the output of one cycle is used as the instruction for the subsequent cycle. (b) The network can be biased towards either R1 and $\mathbf{R 2}$ or $\mathbf{T} \mathbf{1}$ and $\mathbf{T 2}$ by the addition of an instructional template to the initial reaction mixture (in this case, using either $\mathbf{T 1}$ or $\mathbf{R 2}$ ). However, the initial bias in the ratio [RR]/[SR] is eroded over the four subsequent cycles when $20 \mathrm{~mol} \%$ of the output of one cycle is used as the instruction for the subsequent cycle. When the initial bias is provided by $\mathbf{R 2}$ (red bars), $[\mathbf{R R}] /[\mathbf{S R}]=3.75$ after Cycle 0 and 2.54 after Cycle 4. When the initial bias is provided by T1 (blue bars), [RR]/[SR] $=1.14$ after Cycle 0 and 2.55 after Cycle 4. (c) The data from the serial transfer experiments demonstrate that the ratio [RR]/[SR] converges to a single value irrespective of the starting input condition.

After this time, the ratio of reciprocal replicators to self-replicators $([\mathbf{R R}] /[\mathbf{S R}]=$ $([\mathbf{R 1}]+[\mathbf{R 2}]) /([\mathbf{T} 1]+[\mathbf{T} 2]))$ in this sample (Figure 3a, Cycle 0) was 2.06:1. Next, the mixture of products obtained at the end of Cycle 0 was used as an instructional input for Cycle 1. This process simulates pressures on the network arising from compositional changes in the reagent feed and also environmental changes. To this end, $10 \mathrm{~mol} \%$ of the output from Cycle 0 was added to a fresh solution of reagents $\mathbf{A}$ to $\mathbf{D}$ in $\mathrm{CDCl}_{3}$ such that $[\mathbf{A}]=$ $[\mathbf{B}]=[\mathbf{C}]=[\mathbf{D}]=10 \mathrm{mM}$. Once again, the formation of replicators $\mathbf{T 1}, \mathbf{T 2}$, $\mathbf{R} \mathbf{1}$ and $\mathbf{R 2}$ was monitored by $470 \mathrm{MHz}{ }^{19} \mathrm{~F}\left\{{ }^{1} \mathrm{H}\right\}$ NMR spectroscopy. This process was repeated a further two times. The results of these experiments (Figure 3a) reveal that, while the bias of the network towards reciprocal replicators does increase from Cycle $0(2.06: 1)$ to Cycle 3 
(2.30:1), the largest change occurs between Cycle 0 and Cycle 1 (2.26:1). This observation indicates that the relative sizes of the four replicator populations are persistent under these conditions.

In order to probe the stability of the network towards larger perturbations, we next looked at the outcome of the recycling experiments where an instructional template was added in Cycle 0 to generate an initial bias in the replicator populations. In this context, we performed two series of experiments (Figure $3 b$ ) whose basic setup was identical to that described previously. In the first series, a solution of reagents $\mathbf{A}$ to $\mathbf{D}$ in $\mathrm{CDCl}_{3}$ was prepared $([\mathbf{A}]=[\mathbf{B}]=[\mathbf{C}]=[\mathbf{D}]=10 \mathrm{mM}$, Figure $3 \mathrm{~b})$, and reciprocal replicator $\mathbf{R 2}$ was added as an instructional template at a concentration of $2 \mathrm{mM}$ at $t=0$. The addition of this template simulates a large perturbation in the replicator population from an exogenous source. The formation of the minimal replicators, T1 and T2, and the reciprocal replicators, $\mathbf{R} 1$ and $\mathbf{R} 2$ was assayed by $470 \mathrm{MHz}{ }^{19} \mathrm{~F}\left\{{ }^{1} \mathrm{H}\right\}$ NMR spectroscopy after complete consumption of starting materials. After this time, the ratio of reciprocal replicators to self-replicators ([RR]/[SR]) in this sample (Figure 3b, top, Cycle 0) was significantly higher, at 3.75:1, than in the experiment (Figure 3a) where no instructional template had been added. However, as the output of one cycle was used as the instruction for the subsequent cycle, this bias was eroded rapidly and, after Cycle 4, has settled at 2.54:1, a ratio close to the steady state reached in the case of the set of experiments (Figure 3a) that were uninstructed initially.

When this process was repeated with self-replicator $\mathbf{T} 1$ as the initial instruction, the pattern of results was similar. A solution of reagents $\mathbf{A}$ to $\mathbf{D}$ in $\mathbf{C D C l}_{3}$ was prepared ([A] $=[\mathbf{B}]=[\mathbf{C}]=[\mathbf{D}]=10 \mathrm{mM}$, Figure $3 \mathrm{~b}$ ), and self-replicator $\mathbf{T 1}$ was added as an instructional template at a concentration of $2 \mathrm{mM}$ at $t=0$. The final ratio of reciprocal replicators to self-replicators ([RR]/[SR]) in this sample (Figure 3b, bottom, Cycle 0 ) was significantly lower, at 1.14:1, than that in the experiment (Figure 3a) where no instructional template had been added. Once again, as the output of one cycle was used as the instruction for the subsequent cycle-after Cycle 4, the ratio settled at 2.55:1-a value close to the steady state reached in both the case of the recycling experiment (Figure 3a) that was uninstructed initially and that where $\mathbf{R} 2$ was used as the initial instruction. 
These results are striking and demonstrate that this network of replicators possesses a natural, steady state composition (derived from the network fingerprint) and that the network is resistant to changes away from this composition, at least within the confines of a well-stirred batch reactor where there is no continuous ${ }^{19}$ inflow or outflow of material. In order to understand the origin of persistence of this network fingerprint, we employed a series of kinetic simulations using a mass action (Script S3) model based on solving ordinary differential equations (for full details, see Supporting Information, Section S1.2.4) that was capable of reproducing (Figure S8) the trends in the ratio of reciprocal to self-replicators observed in the recycling experiments that we carried out experimentally. The results of these simulations showed that the relative concentrations of the four catalytically-active ternary complexes, namely $[\mathbf{A} \cdot \mathbf{D} \cdot \mathbf{R} 1],[\mathbf{B} \cdot \mathbf{C} \cdot \mathbf{R 2}],[\mathbf{A} \cdot \mathbf{B} \cdot \mathbf{T} 1]$, [C·D·T2] (Figure 1a), and how these concentrations vary with time, plays a critical role in determining the output composition of the network.

Accordingly, we extracted from the simulation data for each cycle the maximum concentration of each ternary complex ([Concentration] $]_{\max }$ ) and the time point in each experiment at which this concentration maximum is achieved for each of the replicating templates $\left(t_{\max }\right)$. When the network is instructed initially with a reciprocal template (either $\mathbf{R} 1$ or R2), the locations of these maxima for the ternary complexes converge rapidly (Figures $4 \mathrm{a}$ and $4 \mathrm{~b}$ ) towards two points in this concentration-time parameter spaceone point for the reciprocal replicators and one point for the self-replicators-as the cycle number increases from 0 to 4 . For example, in the data for the simulation where $\mathbf{R 1}$ is the instructing template (Figure $4 \mathrm{a}$, top left), the concentration maxima for [A·B·T1] and [C·D·T2] are around $25 \mu \mathrm{M}$ and occur close to $20000 \mathrm{~s}$ in Cycle 0 . As the cycle number increases (Figure 4a, blue colored arrows), the maxima for T1 and T2 converge to a point around $60 \mu \mathrm{M}$ at $5500 \mathrm{~s}$. Similarly, the maxima for R1 and R2 converge to a point around $150 \mu \mathrm{M}$ at $5500 \mathrm{~s}$ as the cycle number increases (Figure $4 \mathrm{a}$, red colored arrows). The ratio of these maxima for the ternary complexes $([\mathbf{A} \cdot \mathbf{D} \cdot \mathbf{R} 1]+$ $[\mathbf{B} \cdot \mathbf{C} \cdot \mathbf{R 2}] /[\mathbf{A} \cdot \mathbf{B} \cdot \mathbf{T} 1]+[\mathbf{C} \cdot \mathbf{D} \cdot \mathbf{T 2}]=2.45)$ mirrors that of the final concentrations of the four replicators $(([\mathbf{R} 1]+[\mathbf{R 2}]) /([\mathbf{T 1}]+[\mathbf{T 2}]))$ closely and is essentially invariant after four cycles. Similar convergence is also evident where the network is uninstructed (Figure 4c) or instructed initially with a self-replicating template (either T1 or T2, Figure 4d and 4e). 

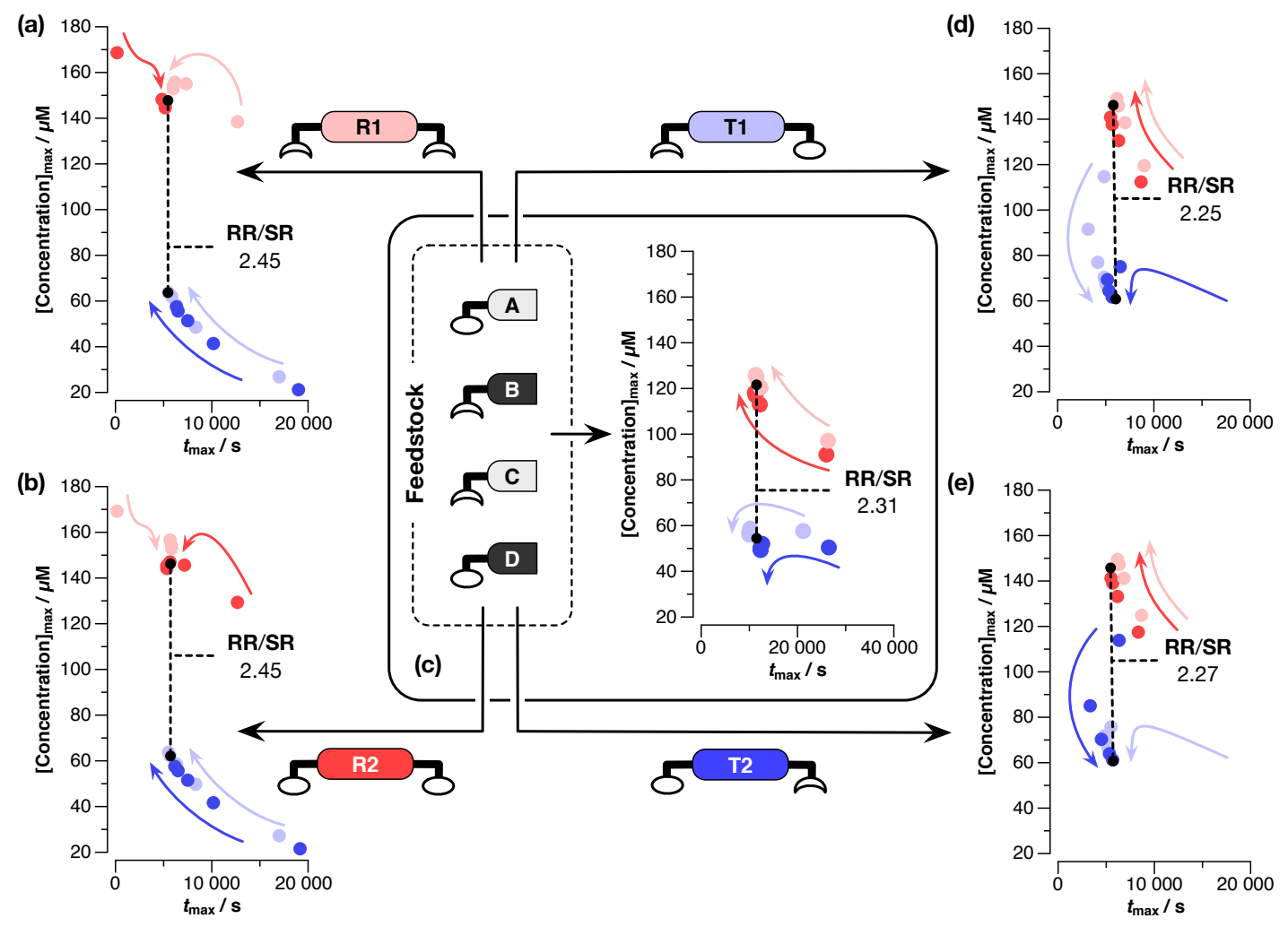

Figure 4. Data from the serial transfer experiments shown in Figure 3 can be reproduced using an appropriate kinetic model (Figure S8, script S3). This kinetic model can be used to extract the behavior of the critical catalytically active ternary complexes, $[\mathbf{A} \cdot \mathbf{D} \cdot \mathbf{R} 1],[\mathbf{B} \cdot \mathbf{C} \cdot \mathbf{R} 2],[\mathbf{A} \cdot \mathbf{B} \cdot \mathbf{T} 1],[\mathbf{C} \cdot \mathbf{D} \cdot \mathbf{T} 2]$, as a function of both cycle number and time. The maximum concentrations of these catalytically-active ternary complexes ([Concentration $\left.]_{\max }\right)$ and the times at which these maxima are reached $\left(t_{\max }\right)$ converge as the cycle number increases when the multicyclic network is (A and $\mathbf{B})$ instructed with reciprocal replicators, (C) un-instructed, and ( $\mathbf{D}$ and $\mathbf{E}$ ) instructed with self-replicators. The colored arrows indicate the paths described by the changes in these parameters for each ternary complex with increasing cycle number. As the system approaches convergence (black points), the composition of the material used as input for the next cycle becomes close to invariant. As a result, RR/SR $(=([\mathbf{A} \cdot \mathbf{D} \cdot \mathbf{R} 1]+[\mathbf{B} \cdot \mathbf{C} \cdot \mathbf{R 2}]) /([\mathbf{A} \cdot \mathbf{B} \cdot \mathbf{T} 1]+$ $[\mathbf{C} \cdot \mathbf{D} \cdot \mathbf{T 2}]))$ tends to a limiting condition and, therefore, the product distribution in subsequent cycles convergences on a single $[\mathbf{R R}] /[\mathbf{S R}]$ slowly.

Once again, the locations of the maxima for the relevant ternary complexes converge rapidly towards two points in the concentration-time parameter space as the cycle number increases from 0 to 4 and the ratio of the maximum concentrations of ternary complexes mirrors closely the ratio of the final concentrations of the four replicators. Once again, the ratios are essentially invariant after four cycles. These results indicate that the catalytic encoding present within this network-its fingerprint defined by the interrelationships between the constituent replicating templates-stabilizes the output of the network. The network is therefore capable of recovering from significant 
perturbation to its composition, thereby preserving the innate composition of this system. The simulations shown in Figure 4 relate to starting conditions in which the concentrations of the starting materials are all equal $(10 \mathrm{mM})$. In order to demonstrate that the persistence observed is a result of the network connectivity as opposed to the specific kinetic and thermodynamic properties of the replicators used, we performed two additional sets of simulations (for full details, see Supporting Information, Section S1.2.4).

In the first set of simulations, we explored ${ }^{20}$ how variations in the concentrations of starting materials $\mathbf{A}$ to $\mathbf{D}$ affected the network output. In Condition I (Figure 5a), the concentration of building block $\mathbf{A}$ is reduced to $1 \mathrm{mM}$, while the starting concentrations of $\mathbf{B}$ to $\mathbf{D}$ remain at $10 \mathrm{mM}$. Despite this drastic change, the network exhibits a trend in persistence that is qualitatively similar to that observed for the network when the starting condition is $[\mathbf{A}]$ to $[\mathbf{D}]=10 \mathrm{mM}$. This pattern is repeated (Condition II, Figure $5 \mathrm{a}$ ) when the concentrations of building blocks $\mathbf{A}$ and $\mathbf{B}$ are both reduced to $5 \mathrm{mM}$.
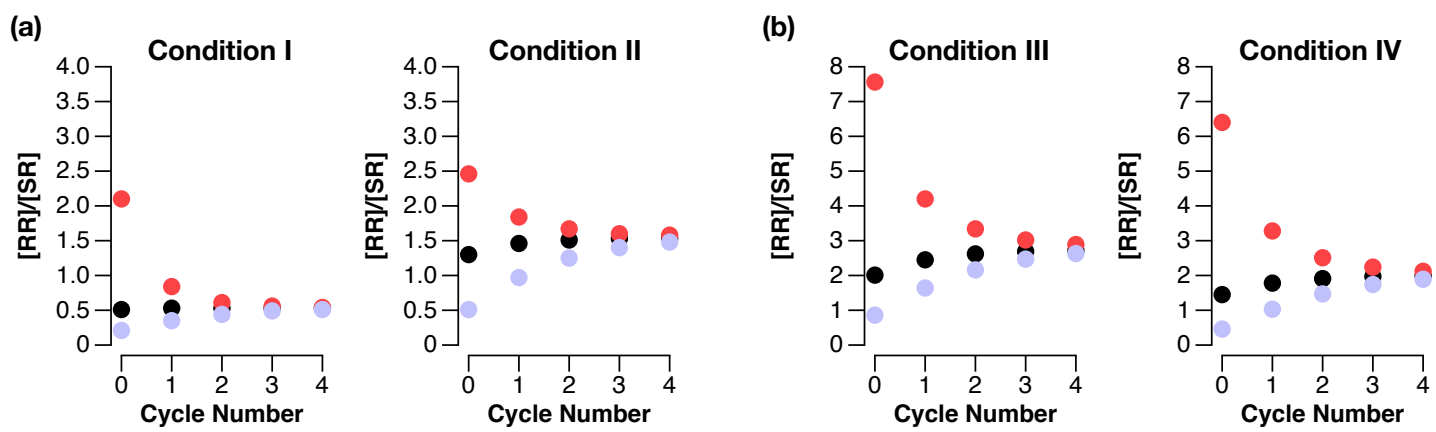

Figure 5. Simulated serial transfer experiments (see Script S3 for the original model) exploring how changes in (a) the initial concentrations of the starting materials $\mathbf{A}$ to $\mathbf{D}$ and (b) in the values of rate and duplex association constants associated with the four template-directed pathways affect the network output. The $[\mathbf{R R}] /[\mathbf{S R}]([\mathbf{R R}]=[\mathbf{R 1}]+[\mathbf{R 2}] ;[\mathbf{S R}]=[\mathbf{T 1}]+[\mathbf{T 2}])$ ratio was simulated as a function of cycle number in the absence of template (black circles) or in the presence of preformed (2 mM) R2 (red circles) or T1 (blue circles) added at $t=0$ in Cycle 0 . The following parameters were changed in simulations I-IV relative to the original simulation (Figure S8) - (a) Condition I: $[\mathbf{A}]=1 \mathrm{mM}$; Condition II: $[\mathbf{A}]=[\mathbf{B}]=5 \mathrm{mM}$; (b) Condition III: $k_{\text {cat }}[\mathbf{C} \cdot \mathbf{D} \cdot \mathbf{T} 2]=1.98 \times 10^{-3} \mathrm{~s}^{-1}, k_{\text {cat }}[\mathbf{B} \cdot \mathbf{C} \cdot \mathbf{R 2}]=2.8 \times 10^{-3} \mathrm{~s}^{-1}$; Condition IV: $[\mathbf{T 1} \cdot \mathbf{T} 1] K_{\mathrm{a}}$ Duplex $=0.2 \times 10^{6} \mathrm{M}^{-1}$, [T2·T2] $K_{\mathrm{a}}^{\text {Duplex }}=20 \times 10^{6} \mathrm{M}^{-1}$, [R1 $\left.\cdot \mathbf{R 2}\right] K_{\mathrm{a}}^{\text {Duplex }}=0.4 \times 10^{6} \mathrm{M}^{-1}$.

In the second set of simulations, we explored ${ }^{20}$ the effect of changes in values of rate and duplex association constants associated with the four template-instructed pathways leading to R1, R2, T1, and T2. In Condition III (Figure 5b), the efficiencies of the catalytic ternary complexes $[\mathbf{C} \cdot \mathbf{D} \cdot \mathbf{T 2}]$ and $[\mathbf{B} \cdot \mathbf{C} \cdot \mathbf{R 2}]$ are increased by a factor of two. 
In Condition IV (Figure 5b), the stabilities of the product duplexes are changed dramatically, by up to $20 \times$. In both cases, the network exhibits trends in persistence that are qualitatively similar to those observed for the network when the original starting conditions are employed (Figure 3).

Taken together, the results of these simulations suggest strongly that the persistence observed experimentally is a direct result of the network connectivity as opposed to the specific kinetic and thermodynamic properties of the replicators used and the starting conditions employed.

\section{Conclusions}

Replicator networks that embed replication processes provide experimental platforms for understanding the appearance on the early Earth of primitive "metabolic" pools capable of processing reagent pools in pre-defined and sustainable ways. Therefore, the establishment and maintenance of networks of replicators possessing connectivities that render them instructable by suitable template inputs can serve as models for these types of processes. In this work, we have created a replicator network whose behavior can be directed specifically by the introduction of instructional templates. Despite the fact that this network responds in predictable ways to instructions provided by these replicating templates, the results presented here show that there are compositional boundaries beyond which the network cannot be pushed by addition of a specific instruction provided by a replicating template. Within the environment of a well-stirred batch reactor, it is the connections between the compounds that make up the network - in terms of both their non-covalent interactions and their auto- and crosscatalytic properties - that encode resistance to changes in the composition of the network. As a consequence, this pool of replicators maintains a level of compositional stability and diversity, which can only be broken by placing the network in an environment where multiple steady states are possible. 


\section{ASSOCIATED CONTENT}

\section{Supporting Information}

Details of sample preparation, NMR analyses, kinetic simulations and scripts.

\section{Author Information}

Present Address (T. K. and D. P.): Department of Chemistry, Northwestern University, 2145 Sheridan Road, Evanston, IL 60208-3113, USA.

Corresponding author email: douglas.philp@northwestern.edu.

\section{ORCID iDs:}

DP: orcid.org/0000-0002-9198-4302

TK: orcid.org/0000-0001-7886-9660

\section{Acknowledgements}

This work was supported by the award of a Postgraduate Studentship from Engineering and Physical Sciences Research Council (EP/K503162/1) to TK and by the University of St Andrews. 


\section{Notes and references}

1. (a) Islam, S.; Powner, M. Prebiotic systems chemistry: complexity overcoming clutter. Chem 2017, 2, 470-501. (b) Peretó, J. Out of fuzzy chemistry: from prebiotic chemistry to metabolic networks. Chem. Soc. Rev. 2012, 41, 5394-5403. (c) Shapiro, R. Small molecule interactions were central to the origin of life. Q. Rev. Biol. 2006, 81, 105-126.

2. (a) Ritson, D. J.; Battilocchio, C.; Ley, S. V.; Sutherland, J. Mimicking the surface and prebiotic chemistry of early Earth using flow chemistry. Nat. Commun. 2018, 9, 1921. (b) Sutherland, J. D. The origin of life-out of the blue. Angew. Chem. Int. Ed. 2016, 55, 104121. (c) Mann, S. The origins of life: old problems, new chemistries. Angew. Chem. Int. Ed. 2013, 52, 155-162. (d) Rauchfuss, H. In: Chemical Evolution and the O rigin of Life; Mitchell, T. N. Ed.; Springer: Berlin, 2008. (e) Luisi, P. L. The emergence of life; Cambridge University Press, 2006.

3. (a) Duim, H.; Otto, S. Towards open-ended evolution in self-replicating molecular systems. Beilstein J. Org. Chem. 2017, 13, 1189-1203. (b) Ashkenasy, G.; Hermans, T. M.; Otto, S; Taylor, A. F. Systems chemistry. Chem. Soc. Rev. 2017, 46, 2543-2554. (c) Miljanić, O. Š. Small-molecule systems chemistry. Chem 2017, 2, 502-524. (d) de la Escosura, A.; Briones, C.; Ruiz-Mirazo, K. The systems perspective at the crossroads between chemistry and biology. J. Theor. Biol. 2015, 381, 11-22. (e) Mattia, E.; Otto, S.; Supramolecular systems chemistry. Nat. Nanotechnol. 2015, 10, 111-119. (f) RuizMirazo, K.; Briones, C.; de la Escosura, A. Prebiotic systems chemistry: new perspectives for the origins of life. Chem. Rev. 2014, 114, 285-366.

4. (a) Tirard, S. Origin of life and definition of life, from Buffon to Oparin. Origins Life Evol. Biospheres 2010, 40, 215-220. (b) Benner, S. A. Defining life. Astrobiology 2010, 10, 1021-1030. (c) Lazcano, A. What is life? Chem. Biodiversity 2008, 5, 1-15. (d) Cleland, C. E.; Chyba, C. F. Defining 'life'. Orig. Life Evol. Biospheres 2002, 32, 387-393. (e) Joyce, G. F. Foreword. In Origins of Life: the Central Concepts, pp. xi-xii; Deamer, D. W.; Fleischaker, G. Eds.; Jones and Bartlettt, Boston, 1994. (f) Schrödinger, E. What is life? Cambridge University Press, Cambridge, 1944.

5. (a) Kosikova, T.; Philp, D. Exploring the emergence of complexity using synthetic replicators. Chem. Soc. Rev. 2017, 46, 7274-7305. (b) Bissette, A. J.; Fletcher, S. P. 
Mechanisms of autocatalysis. Angew. Chem. Int. Ed. 2013, 52, 12800-12826. (c) Philp, D.; Huck, J. Supramolecular Chemistry: From Molecules to Nanomaterials; John Wiley \& Sons, Ltd.: New York, 2012; Vol. 4, pp. 1415-1445. (d) Vidonne, A.; Philp, D. Making molecules make themselves - the chemistry of artificial replicators. Eur. J. Org. Chem. 2009, 593-610. (e) Dadon, Z.; Wagner, N.; Ashkenasy, G. The road to non-enzymatic molecular networks. Angew. Chem. Int. Ed. 2008, 47, 6128-6136. (f) Patzke, V.; von Kiedrowski, G. Self-replicating systems. ARKIVOC 2007, 46, 293-310. (g) Paul, N.; Joyce, G. F. Minimal self-replicating systems. Curr. Opin. Chem. Biol. 2004, 8, 634-639. (h) Von Kiedrowski, G. Minimal replicator theory I: parabolic versus exponential growth. Bioorganic Chem. Front. 1993, 3, 113-146.

6. (a) Robertson, C. C.; Kosikova, T.; Philp, D. An environmentally responsive reciprocal replicating network. J. Am. Chem. Soc. 2018, 140, 6832-6841. (b) Sadownik, J. W.; Kosikova, T.; Philp, D. Generating system-level responses from a network of simple synthetic replicators. J. Am. Chem. Soc. 2017, 139, 17565-17573. (c) Kosikova, T.; Philp, D. A critical cross-catalytic relationship determines the outcome of competition in a replicator network. J. Am. Chem. Soc. 2017, 139, 12579-12590. (d) Bottero, I.; Huck, J.; Kosikova, T.; Philp, D. A synthetic replicator drives a propagating reaction-diffusion front. J. Am. Chem. Soc. 2016, 138, 6723-6726. (e) Kosikova, T.; Hassan, N. I.; Cordes, D. B.; Slawin, A. M. Z.; Philp, D. Orthogonal recognition processes drive the assembly and replication of a [2]rotaxane. J. Am. Chem. Soc. 2015, 137, 16074-16083. (f) Kassianidis, E.; Philp, D. Design and implementation of a highly selective minimal self-replicating system. Angew. Chem. Int. Ed. 2006, 45, 6344-6348.

7. For examples of synthetic replicating systems based on oligonucleotides, see: (a) Plöger, T. A.; von Kiedrowski, G. A self-replicating peptide nucleic acid. Org. Biomol. Chem. 2014, 12, 6908-6914. (b) Lincoln, T. A.; Joyce, G. F. Self-sustained replication of an RNA enzyme. Science 2009, 323, 1229-1232. (c) Paul, N.; Joyce, G. F. A self-replicating ligase ribozyme. Proc. Natl. Acad. Sci. USA 2002, 99, 12733-12740. (d) von Kiedrowski, G.; Wlotzka, B.; Helbing, J.; Matzen, M.; Jordan, S. Parabolic growth of a self-replicating hexadeoxynucleotide bearing a 3'-5'-phosphoamidate linkage. Angew. Chem. Int. Ed. Engl. 1991, 30, 423-426. (e) von Kiedrowski, G. A self-replicating hexadeoxynucleotide. Angew. Chem. Int. Ed. Engl. 1986, 25, 932-935. 
8. For examples of synthetic replicating systems based on peptides, see: (a) Altay, M.; Altay, Y.; Otto, S. Parasitic behavior of self-replicating molecules. Angew. Chem. Int. Ed. 2018, 139, 10564-10568. (b) Sadownik, J. W.; Mattia, E.; Nowak, P.; Otto, S. Diversification of self-replicating molecules. Nature Chem. 2016, 8, 264-269. (c) Colomb-Delsuc, M.; Mattia, E.; Sadownik, J. W.; Otto, S. Exponential self-replication enabled through a fibre elongation/breakage mechanism. Nat. Commun. 2015, 6, 7427. (d) Dadon, Z.; Wagner, N.; Alasibi, S.; Samiappan, M.; Mukherjee, R.; Ashkenasy, G. Competition and cooperation in dynamic replication networks. Chem. Eur. J. 2015, 21, 648-654. (e) Samiappan, M.; Dadon, Z.; Ashkenasy, G. Replication NAND gate with light as input and output. Chem. Commun. 2011, 47, 710-712. (f) Carnall, J. M. A.; Waudby, C. A.; Belenguer, A. M.; Stuart, M. C. A.; Peyralans, J. J. -P.; Otto, S. Mechanosensitive selfreplication driven by self-organization. Science 2010, 327, 1502-1506. (g) Issac, R.; Chmielewski, J. Approaching exponential growth with a self-replicating peptide. J. Am. Chem. Soc. 2002, 124, 6808-6809. (h) Saghatelian, A.; Yokobayashi, Y.; Soltani, K.; Ghadiri, M. R. A chiroselective peptide replicator. Nature 2001, 409, 797-801. (i) Lee, D. H.; Granja, J. R.; Martinez, J. A.; Severin, K.; Ghadiri, M. R. A self-replicating peptide. Nature 1996, 382, 525-528.

9. For examples of synthetic replicating systems based on small organic molecules, see: (a) Morrow, S. M.; Colomer, I.; Fletcher, S. P. A chemically fueled self-replicator. Nat. Commun. 2019, 10, 1011. (b) Colomer, I.; Morrow, S. M.; Fletcher, S. P. A transient selfassembling self-replicator. Nat. Commun. 2018, 9, 2239. (c) Taylor, J. W.; Eghtesadi, S. A.; Points, L. J.; Cronin, L. Autonomous model protocell division driven by molecular replication. Nat. Commun. 2017, 8, 237. (d) Kindermann, M.; Stahl, I.; Reimold, M.; Pankau, W. M.; von Kiedrowski, G. Systems chemistry: kinetic and computational analysis of a nearly exponential organic replicator. Angew. Chem. Int. Ed. 2005, 44, 67506755. (e) Wang, B.; Sutherland, I. O. Self-replication in a Diels-Alder reaction. Chem. Commun. 1997, 1495-1496. (f) Rotello, V.; Hong, J. -I.; Rebek, J. Jr. Sigmoidal growth in a self-replicating system. J. Am. Chem. Soc. 1991, 113, 9422-9423.

10. Sadownik, J. W.; Philp, D. A simple synthetic replicator amplifies itself from a dynamic reagent pool. Angew. Chem. Int. Ed. 2008, 47, 9965-9970. 
11. (a) Helwig, B.; van Sluijs, B.; Pogodaev, A. A.; Postma, S. G. J.; Huck, W. T. S. Bottomup construction of an adaptive enzymatic reaction-network. Angew. Chem. Int. Ed. 2018, 57, 14065-14069. (b) Ashkenasy, G.; Dadon, Z.; Alesebi, S.; Wagner, N.; Ashkenasy, N. Building logic into peptide networks: bottom-up and top-down. Isr. J. Chem. 2011, 51, 106-117.

12. Postma, S. G. J.; te Brinke, D.; Vialshin, I. N.; Wong, A. S. Y.; Huck, W. T. S. A trypsinbased bistable switch. Tetrahedron 2017, 73, 4896-4900.

13. (a) Pogodaev, A. A.; Wong, A. S. Y.; Huck, W. T. S. Photochemical control over oscillations in chemical reaction networks. J. Am. Chem. Soc. 2017, 139, 15296-15299. (b) Semenov, S. N.; Kraft, L. J.; Ainla, A.; Zhao, M.; Baghbanzadeh, M.; Campbell, V. E.; Kang, K.; Fox, J. M.; Whitesides, G. M. Autocatalytic, bistable, oscillatory networks of biologically relevant organic reactions. Nature 2016, 537, 656-660. (c) Wagner, N.; Alasibi, S.; Peacock-Lopez, E.; Ashkenasy, G. Coupled oscillations and circadian rhythms in molecular replication networks. J. Phys. Chem. Lett. 2015, 6, 60-65. (d) Fuji, T.; Rondelez, Y. Predator-prey molecular ecosystems. ACS Nano 2013, 7, 27-34. (e) Montagne, K.; Plasson, R.; Sakai, Y.; Fujii, T.; Rondelez, Y. Programming an in vitro DNA oscillator using a molecular networking strategy. Mol. Syst. Biol. 2011, 7:466.

14. (a) Skorb, E. V.; Semenov, S. N. Mathematical analysis of a prototypical autocatalytic reaction network. Life 2019, 9, 42. (b) Hordijk, W.; Steel, M.; Dittrich, P. Autocatalytic sets and chemical organizations: modelling self-sustaining reaction networks at the origin of life. (c) Yeates, J. A. M.; Hilbe, C.; Zwick, M.; Nowak, M. A.; Lehman, N. Dynamics of prebiotic RNA reproduction illuminated by chemical game theory. Proc. Natl. Acad. Sci. USA 2016, 113, 5030-5035. (d) Vasas, V.; Szathmáry, Santos, M. Lack of evolvability in self-sustaining autocatalytic networks constraints metabolism-first scenarios for the origin of life. Proc. Natl. Acad. Sci. USA 2010, 107, 1470-1475. (e) Hordijk, W.; Hein, J.; Steel, M. Autocatalytic sets and the origin of life. Entropy 2010, 12, 1733-1742.

15. (a) Kassianidis, E.; Pearson, R. J.; Wood, E. A.; Philp, D. Designing instructable networks using synthetic replicators. Faraday Discuss. 2010, 145, 235-254. (b) Kassianidis, E.; Philp, D. Reciprocal template effects in a simple synthetic system. Chem. Commun. 2006, 4072-4074. 
16. The adjective "system-level", when used in conjunction with nouns such as "behavior", "effect" or "properties", is used to represent effects that arise as a result of the presence of interactions and reactions within the system under study. These indirect connections between members in a network result in the observation of effects that are not expressed in the absence of these catalytic and interactional inter-relationships.

17. Becker, S.; Schneider, C.; Okamura, H.; Crisp, A.; Amatov, T.; Dejmek, M.; Carell, T.; Wet-dry cycles enable the parallel origin of canonical and non-canonical nucleosides by continuous synthesis. Nat. Commun. 2018, 9, 163.

18. (a) Hordijk, W.; Vaidya, N.; Lehman, N. Serial transfer can aid the evolution of autocatalytic sets. J. Syst. Chem. 2014, 5, 4. (b) Joyce, G. F. Evolution in an RNA world. Cold Spring Harb. Symp. Quant. Biol. 2009, 74, 17-23.

19. Wagner, N.; Hochberg, D.; Peacock-Lopez, E.; Maity, I.; Ashkenasy, G. Open prebiotic environments drive emergent phenomena and complex behaviour. Life 2019, 9, 45.

20. For detailed descriptions of the additional simulations exploring how changes in the initial concentrations of the starting materials $\mathbf{A}$ to $\mathbf{D}$ and in the values of rate and duplex association constants associated with the four template-directed pathways affect the network output, see Figure S9, Figure S10 and Table S1. 


\section{For ToC Graphic only:}

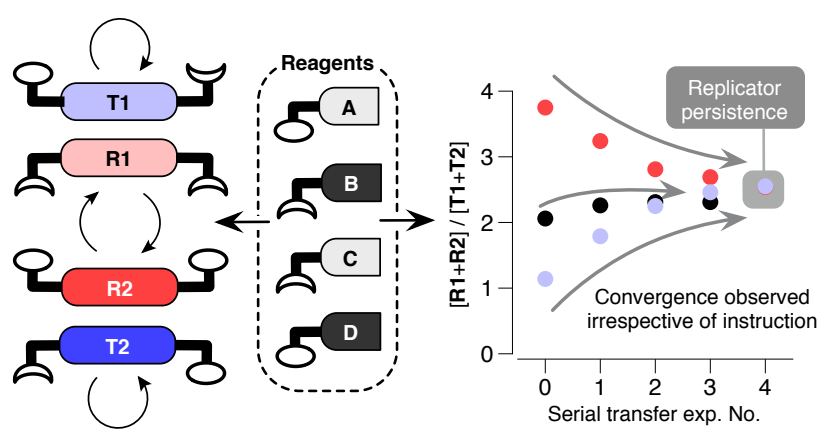

